\title{
Research Article \\ The Use of Fullerene C60 to Preserve Testicular Tissue after Cryopreservation
}

\author{
Nataliia Volkova (D), Mariia Yukhta (D), and Anatoliy Goltsev \\ Department of Cryopathophysiology and Immunology, Institute for Problems of Cryobiology and Cryomedicine of the National \\ Academy of Sciences of Ukraine, Pereyaslavskaya Str., 23, Kharkov 61015, Ukraine
}

Correspondence should be addressed to Nataliia Volkova; volkovana781@gmail.com

Received 20 January 2021; Revised 22 March 2021; Accepted 31 March 2021; Published 17 April 2021

Academic Editor: Nicholas Dunne

Copyright (c) 2021 Nataliia Volkova et al. This is an open access article distributed under the Creative Commons Attribution License, which permits unrestricted use, distribution, and reproduction in any medium, provided the original work is properly cited.

\begin{abstract}
Autologous transplantation of cryopreserved fragments of an immature testis is an actively developing approach to save fertility in patients facing a gonadotoxic therapy. The use of bioavailable fullerene C60 as a powerful antioxidant opens up a new potential for the prevention and correction of ischemic-reperfusion pathological processes in tissues including those associated with freezingthawing procedure. In this work, we aimed to study the antioxidant status, apoptotic/necrotic processes, and morphological characteristics of cryopreserved fragments of the seminiferous tubules of testis (CrFSTT) of immature rats after incubation in media with different concentrations of fullerene C60 $(10,15$, and $20 \mu \mathrm{g} / \mathrm{mL})$. Our results indicated that the addition of C60 in a concentration of $15 \mu \mathrm{g} / \mathrm{mL}$ decreased ROS production, cytochrom $\mathrm{C}$ release, and degree of histological damage of spermatogenic epithelium as well as increased the activity of the mitochondria, antioxidant defense system, and cell density in histological sections of CrFSTT compared to the control. Fullerene C60 at investigated concentrations did not impact significantly on apoptosis in cells of CrFSTT but, after incubation with $15 \mu \mathrm{g} / \mathrm{mL}$ C60, a percentage of living cells was 1.2 -fold higher and a value of necrotic ones in this group was 1.6 -fold lower than the control samples $(p<0.05)$. Relative amount of cells of the spermatogonia germ layer did not differ between the studied concentrations. The general analysis of obtained data showed that the C60 addition in the concentration of $15 \mu \mathrm{g} / \mathrm{mL}$ was the most optimal for the rehabilitation of CrFSTT. The results can be used for the development of an effective rehabilitation medium for the cryopreserved testicular tissue.
\end{abstract}

\section{Introduction}

Currently, an autologous transplantation of cryopreserved fragments of an immature testis is an actively developing approach to restore fertility of adolescent patients facing gonadotoxic therapy [1]. The advantage of this method is a preservation of spermatogenic cell niche, due to the support of cellular contacts and interactions, which contributes to the normal development of germ cells. But there are other, no less difficult tasks: minimization of tissue damage by ice crystals and avoidance of ischemic-reperfusion pathological processes associated with cryopreservation.

Some properties of nanoparticles can be useful in solving these problems. For example, oxidized quasicarbon nitride quantum dots can inhibit ice growth/recrystallization through the density of hydrogen bonds formed with ice [2].
And nanoparticle-mediated trehalose delivery technology helps to overcome the major limitation of ultralow permeability of this disaccharide resulting in favorable postthaw cell survival rates without the need of any organic solvent [3].

But the capabilities of nanoparticles are not limited to freeze-thaw processes, because they can also be used for the rehabilitation of biological objects after cryopreservation. Since oxidative stress is one of the cryodamage factors, the use of carbon nanoparticles as components of rehabilitation media is a promising direction for the cell and tissue regeneration after cryopreservation. So, the idea of our study is to increase the efficiency of cryopreservation of seminiferous tubules using water-soluble fullerene C60 as an antioxidant agent.

Fullerene C60 has unique structural and functional properties, including nanosize, low surface energy, high chemical 
stability, spherical shape, weak intermolecular bonding, and high load-bearing capacity [4]. The C60 molecule is able to interact with biological membranes, penetrating into the intracellular space by passive diffusion or endocytosis [5].

Previous biological in vitro and in vivo screening of unmodified fullerene C60 aqueous colloid solution showed that it does not have acute toxic effects on normal cells at low concentrations, is not immunogenic, is nonallergenic, and is able to neutralize the excess of free radicals $[6,7]$. Thus, the use of bioavailable fullerene $\mathrm{C} 60$ as a powerful antioxidant opens up a new potential for the prevention and correction of ischemic-reperfusion pathological processes in tissues associated with the freezing-thawing procedure.

Here, we present a study on how different concentrations of fullerene C60 affect the antioxidant status, apoptotic/necrotic processes, and morphological characteristics of cryopreserved fragments of the seminiferous tubules of the testis (CrFSTT) of immature rats.

\section{Materials and Methods}

2.1. Animals. All the manipulations were carried out in accordance to the European convention for the protection of vertebrate animals used for experimental and other scientific purposes (Strasbourg, 18.III.1986). The protocols were approved by the Bioethics Committee of Institute for Problems of Cryobiology and Cryomedicine of the NAS of Ukraine (permit no. 2016-05).

Outbreed white sexually immature male rats (aged 7-8 weeks, $n=50$ ) were used in the study. Humane euthanasia by $\mathrm{CO}_{2}$ asphyxiation was performed to obtain testes of experimental animals right before the study. Excised testes were rinsed in L-15 medium (Leibovitz) (prod. no. L0300500, BioWest, Nuaille, France). Tunica albuginea was removed using sterile medical instruments, and the testes were then rinsed again. Fragments of the seminiferous tubules of the testis weighing $75 \pm 5 \mathrm{mg}$ were isolated mechanically and cryopreserved.

2.2. Cryopreservation. Cryoprotective medium based on a fibrin gel with the addition of glycerol (prod. no. G5516, Sigma-Aldrich, St. Louis, USA) at the final concentration of $0.7 \mathrm{M}$ was made ex tempore. Fibrin gel was received from the fresh blood of animals, which was obtained from a cardiac vein and centrifuged for $12 \mathrm{~min}$ at a rate of $1000 \mathrm{~g}$. The tissue fragments were transferred into $2 \mathrm{~mL}$ TPP $^{\circledR}$ cryotubes (prod. no. Z760951, Sigma-Aldrich, St. Louis, USA), where they were exposed to the cryoprotective medium for $30 \mathrm{~min}$ at $4^{\circ} \mathrm{C}$ [8]. Then, cryotubes with samples were cooled in vapors of liquid nitrogen for 40 min down to $-70^{\circ} \mathrm{C}$ and transferred to liquid nitrogen $\left(-196^{\circ} \mathrm{C}\right)$ [9]. Cryotubes were stored in a cryobank for a month and then were warmed up in a water bath at $40^{\circ} \mathrm{C}$ until a liquid phase appearance. After thawing, cryoprotectants were removed from the samples by a threestep change of the solution to the L-15 medium.

2.3. Manipulations with Fullerene C60. Fullerene- $\mathrm{C}_{60}$ (99.9\%) was purchased from Sigma-Aldrich, St. Louis, USA (prod. no. 572500). It consisted of agglomerates of spherical or fibrous primary particles. To obtain an aqueous suspension of fullerene C60, a method based on the transfer of this carbon nanostructure from toluene to water followed by sonication was used [10]. The result was a typical colloid solution of C60.

The thawed CrFSTT were incubated at $22^{\circ} \mathrm{C}$ for $30 \mathrm{~min}$ in the L-15 medium with the addition of fullerene $\mathrm{C} 60$ at final concentrations of 10,15 , and $20 \mu \mathrm{g} / \mathrm{mL}$. The samples incubated in the L-15 medium without C60 served as a control.

The scheme of experiment is shown in Figure 1.

2.4. Analysis of Total Antioxidant Status (TAS). The samples of CrFSTT were homogenized, filtered, and centrifugated (1000 $\mathrm{g}$ for $10 \mathrm{~min}$ ). TAS activity was estimated quantitatively by the method of UV spectrophotometry (ERBA CHEM 7, Erba Lachema s.r.o., Brno, Czech Republic) using test kits (prod. no. NX2332, Randox Laboratories Ltd., Crumlin, UK) according to the manufacturer's instructions and normalized to $1 \mathrm{mg}$ of protein (prod. no. TP8336, Randox Laboratories Ltd., Crumlin, UK).

2.5. Flowcytometry Analysis. The cell suspension was obtained from CrFSTT by enzymatic disaggregation: the samples were incubated with $0.25 \%$ trypsin $\left(37^{\circ} \mathrm{C}\right.$ for $30 \mathrm{~min}$ ), filtered, and centrifugated (1000 g for $10 \mathrm{~min}$ ).

Intracellular reactive oxygen species (ROS) was estimated quantitatively using a fluorometric test kit (prod. no. MAK142, Sigma-Aldrich, St. Louis, USA). In brief, the cells of CrFSTT $(0.5 \mathrm{~mL})$ at a concentration of $1 \times 10^{6}$ cells $/ \mathrm{mL}$ were suspended with $1 \mu \mathrm{L}$ of ROS detection reagent stock solution and incubated under $5 \% \mathrm{CO}_{2}$ at $37^{\circ} \mathrm{C}$ for one hour.

The apoptotic/necrotic processes in cells of CrFSTT were studied using Annexin-V-FITC (Annexin V) (prod. no. 556419 BD Pharmingen ${ }^{\mathrm{TM}}$, San Jose, USA) and 7aminoactinomycin D (7AAD) (prod. no. 559925, BD Pharmingen $^{\mathrm{TM}}$, San Jose, USA) dyes. The cells of CrFSTT $(0.5 \mathrm{~mL})$ at a concentration of $1 \times 10^{6}$ cells $/ \mathrm{mL}$ were suspended with $5 \mu \mathrm{L}$ of Annexin $\mathrm{V}$ and $5 \mu \mathrm{L}$ of 7AAD. The samples were incubated at $22^{\circ} \mathrm{C}$ for $20 \mathrm{~min}$.

The functional state of the mitochondria was investigated using a mitochondrial membrane potential detection kit JC-1 (prod. no. 551302, BD Pharmingen ${ }^{\mathrm{TM}}$, San Jose, USA). Incubation of the cells with the JC-1 working solution $(0.5 \mathrm{~mL} / \mathrm{sample})$ for $15 \mathrm{~min}$ at $37^{\circ} \mathrm{C}$ in a $\mathrm{CO}_{2}$ incubator was performed. Then, cells were washed twice.

The number of cells positively stained for cytochrome C was determined using the test system Cyt.C (prod. no. 560263 , BD Pharmingen ${ }^{\mathrm{TM}}$, San Jose, USA). The cells of CrFSTT $(0.5 \mathrm{~mL})$ at a concentration of $1 \times 10^{6}$ cells $/ \mathrm{mL}$ suspended with $5 \mu \mathrm{L}$ of Cyt.C and incubated at $22^{\circ} \mathrm{C}$ for $20 \mathrm{~min}$.

All investigations were performed according to the manufacturer's instructions. BD FACSCalibur ${ }^{\mathrm{TM}}$ (Becton Dickinson, San Jose, USA) was used for these tests. Data were analyzed using WinMDI v.2.8.

2.6. Histomorphology. The samples of CrFSTT for histological examination were fixed in $10 \%$ formalin solution (prod. no. HT501128, Sigma-Aldrich, St. Louis, USA). Sections 


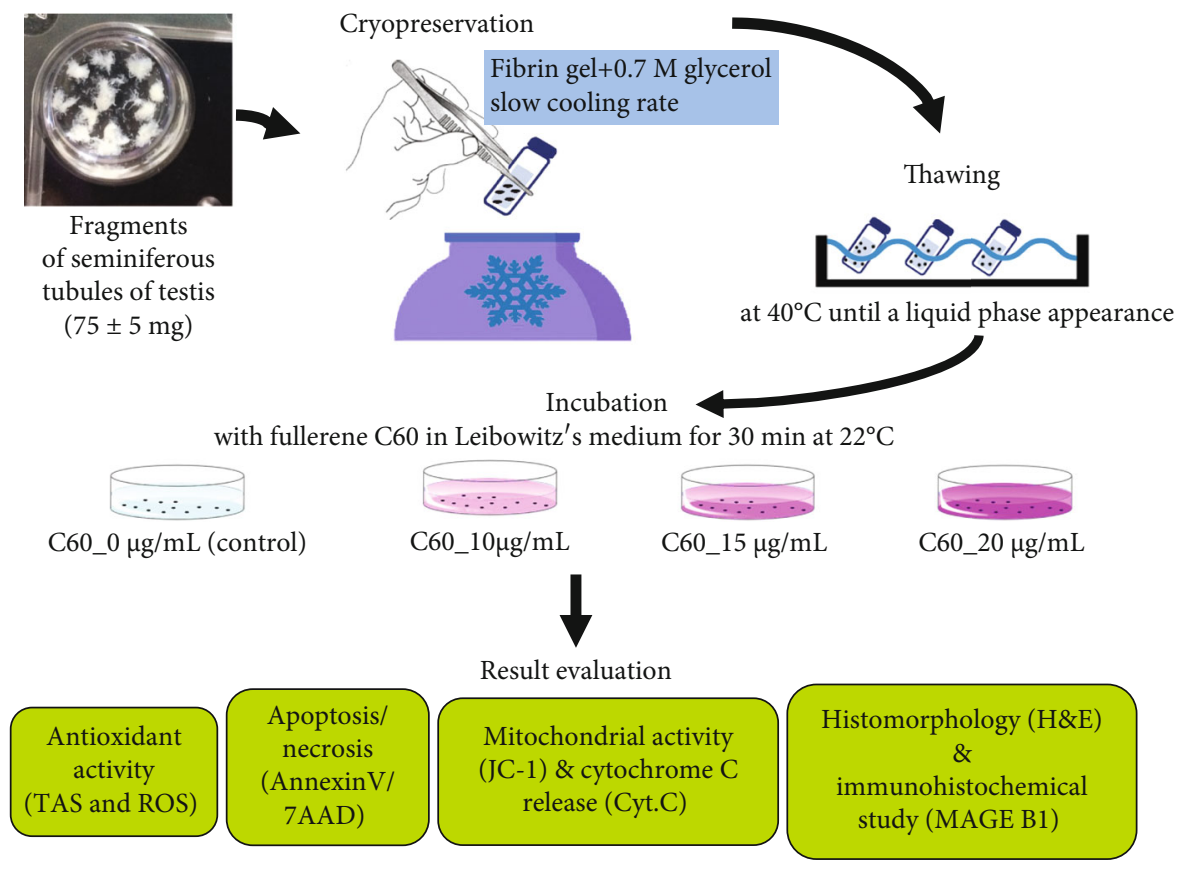

FIgURE 1: Experimental scheme.

were cut from paraffin-embedded blocks ( $7 \mu \mathrm{m}$ thick), stained with hematoxylin and eosin, and studied using Zeiss Axio Observer Z1 inverted microscope (Carl Zeiss Microscopy $\mathrm{GmbH}$, Jena, Germany). Obtained images were processed using ZEISS ZEN 2 (blue edition) (Carl Zeiss Microscopy GmbH, Jena, Germany). Histomorphology was carried out maintaining blinding by involving the third person who did not take part in the experiment. The integrity and the structural changes of spermatogenic epithelium in the fragments of the semeniferous tubules of the testis sections were evaluated semiquantitatively. They were scored as follows: (i) retraction of spermatogenic epithelium cells scored as 0 if absent, as 1 if slight, and as 2 if more obvious; (ii) nuclei condensation was scored as 0 if absent or present in only 1 nucleus, as 1 if $40 \%$ of nuclei were condensed, and as 2 if $40 \%$ were pyknotic; (iii) detachment of cells from the basement membrane was scored as 0 if absent, as 1 if partial, and as 2 if total or observed on $75 \%$ of the circumference; and (iiii) gap formation and shrinkage were scored as 0 if absent, as 1 if slight, and as 2 if more obvious. Therefore, the total score for each section of the seminiferous tubules was between 0 and 8 . It was determined in 30 seminiferous tubules with the calculation of the average value for each observation. The minimal total score (0-1) corresponded to the intact structure of the spermatogenic epithelium. A score more than 1 to 3 points inclusively was accepted as mild damage of spermatogenic epithelium, more than 3 to 6 points inclusively as moderately expressed ones, and in the case of more than 6 points, changes were considered pronounced. An average cell density of the spermatogenic epithelium ( $a$ number of nuclei per area unit) was also evaluated.

2.7. Immunohistochemical Study. Immunohistochemical staining of deparaffinized sections of CrFSTT was performed using polyclonal antibodies to Melanoma Antigen Family B1 (MAGE-B1, prod. no. PA5-51532, 1:500, Invitrogen ${ }^{\mathrm{TM}}$, New York, USA) according to the protocol recommended for the peroxidase detection system Ultra Vision Quantro HRP DAB (prod. no. TL-060-QHD, Thermo Fisher Scientific, Fremont, USA). We counted MAGE-B1 ${ }^{+}$cells using ZEISS ZEN 2 (blue edition) (Carl Zeiss Microscopy GmbH, Jena, Germany) and determined their relative amount as the ratio of the number of stained cells to the total number of cells in the tubule section, which was taken as $100 \%$. For the negative control, MAGE-B1 was replaced by a mouse immunoglobulin G isotype control (prod. no. 02-6502; Invitrogen ${ }^{\mathrm{TM}}$, New York, USA).

2.8. Statistical Analysis. Kruskal-Wallis ANOVA test and multiple comparisons $p$ values were applied to compare the difference between the groups using Statistica 8 (StatSoft Inc., Tulsa, USA) software. Histological scores were compared using a Mann-Whitney $U$-test.

\section{Results and Discussion}

3.1. Effect of Fullerene C60 on TAS and ROS Generation in the CrFSTT. The obtained results showed a 1.5- and 1.7-fold increase $(p<0.05)$ of TAS activity and decreased content of ROS + cells in CrFSTT by 1.2 and 1.3 times $(p<0.05)$, respectively, after C60 addition at concentrations of 10 and $15 \mu \mathrm{g} / \mathrm{mL}$ versus control (Figures 2(a) and 2(b)). The use of $20 \mu \mathrm{g} / \mathrm{mL}$ fullerene C60 did not lead to changes in studied parameters relative to the control and as a result had a significant difference with the previous two concentrations. Thus, the addition of C60 (10 and $15 \mu \mathrm{g} / \mathrm{mL})$ to the incubation medium decreased the ROS generation and increase TAS activity in the CrFSTT. 


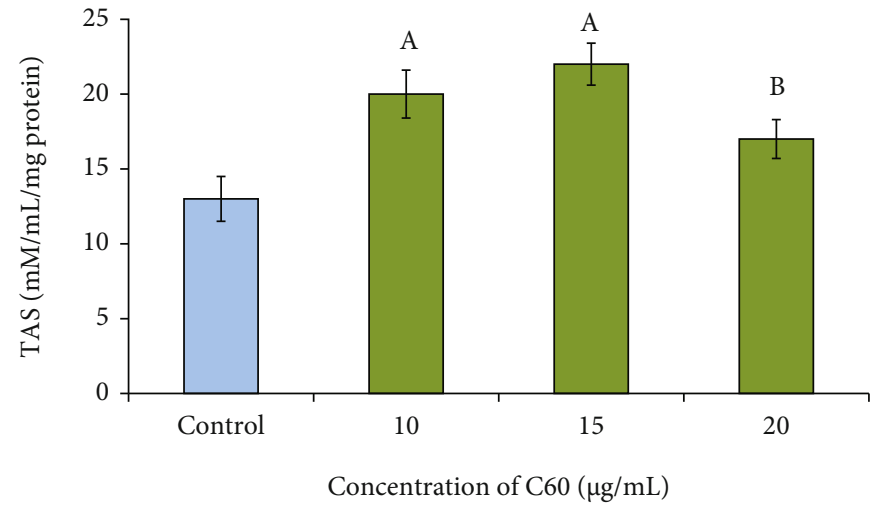

(a)

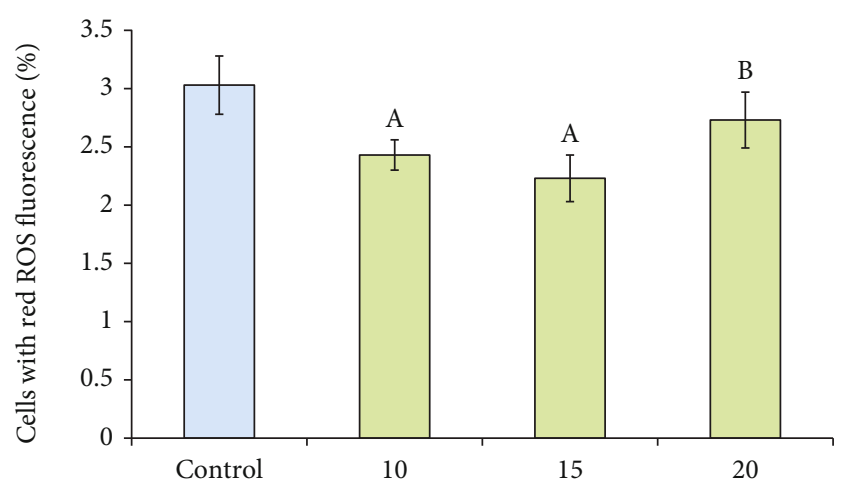

Concentration of C60 $(\mu \mathrm{g} / \mathrm{mL})$

(b)

FIgURE 2: The effect of incubation in the media with C60 addition on TAS activity (a) and content of ROS ${ }^{+}$cells (b) in CrFSTT. Notes: A difference is significant versus the control $(p<0.05)$; ${ }^{\text {B }}$ difference is significant versus C60_15 $\mu \mathrm{g} / \mathrm{mL}(p<0.05)$.

It is known that oxidative stress occurs in response to damage, when the antioxidant activity and absorption capacity of a biological object cannot neutralize free radicals caused by a harmful effect. ROS are involved in different pathophysiological processes in the testis; as a result, oxidative stress causes a testicular dysfunction due to inhibition of steroidogenesis and vasomotion [11]. The main antioxidant enzymes that neutralize the ROS in semen and various parts of the male reproductive system are superoxide dismutase, glutathione peroxidase, and catalase. But, many others so-called "indirect" antioxidant enzymes are also associated with the mechanism of antioxidative protection because they promote the biosynthesis/recycling of thiols or contribute to excretion of oxidized metabolites [12]. At the same time, the increase of TAS activity after the use of C60 at concentrations of 10 and $15 \mu \mathrm{g} / \mathrm{mL}$ is apparently associated with the direct antioxidant action of fullerenes. Due to the presence on the surface of a system of $\pi$-conjugated double bonds between hexa- and pentagonal structures, fullerenes collect and bind free radicals to each other, while they themselves remain unchanged [13]. Thus, the addition of 10 and $15 \mu \mathrm{g} / \mathrm{mL}$ C60 to the rehabilitation medium helps to neutralize oxidative stress in CrFSTT triggered by cryopreservation without significant difference between these two concentrations.

\subsection{Effect of C60 on Apoptotic and Necrotic Processes in} CrFSTT of Rats. The results obtained by flow cytometry (Table 1) suggested that $\mathrm{C} 60$ at all investigated concentrations did not cause the development of necrosis/apoptosis in cells. Conversely, C60 addition at the concentration of $15 \mu \mathrm{g} / \mathrm{mL}$ led to the 1.2 -fold increase $(p<0.05)$ of percentage of live cells (Annexin $\mathrm{V}^{-} / 7 \mathrm{AAD}^{-}$) and to the 1.6-fold decrease $(p<0.05)$ in the number of necrotic cells $\left(\right.$ AnnexinV $\mathrm{V}^{+} / 7 \mathrm{AAD}^{+}$and Annexin $\mathrm{V}^{-} / 7 \mathrm{AAD}^{+}$) compared to the control samples.

The processes for initiating apoptosis are identical for most of the cells. Wherein changes of plasma membrane are observed, phosphatidyl serine goes from the cytoplasmatic part of the bilayer to the outer one, leading to the activation of caspase cascade, chromatin condensation, dysfunction of the mitochondria, and ultimately stopping ATP synthesis. Thus, programmed cell death can be caused by receptor-mediated physiological stimuli due to genetic disorders, physical or chemical triggers, and other changes in cells [14].

The next stage of the study was the quantitative evaluation of the mitochondrial activity and cytochrome $\mathrm{C}$ release in cells of CrFSTT after incubation with C60. The results obtained by flow cytometry (Figures 3(a) and 3(b)) showed that $\mathrm{C} 60$ addition in concentrations of 10 and $20 \mu \mathrm{g} / \mathrm{mL}$ did not affect investigated indexes. The use of C60 in concentration of $15 \mu \mathrm{g} / \mathrm{mL}$ led to a 1.15 -fold increase $(p<0.05)$ in the content of $\mathrm{JC}^{+}$cells (orange fluorescence) and to a 1.3 -fold decrease $(p<0.05)$ in the number of Cyt. $\mathrm{C}^{+}$cells compared to the control samples.

The analysis of the results showed a decrease in the release of cytochrome $\mathrm{C}$ into the cytosol and a high mitochondrial activity in the CrFSTT cells, which had the highest viability index by staining with Annexin $\mathrm{V}$ and 7AAD (Table 1, C60_15 $\mu \mathrm{g} / \mathrm{mL}$ ). As known, the mitochondria play a major role in cellular partitioning of deathregulating signals; the loss of mitochondrial membrane potential is an early event in several types of apoptosis. The high transmembrane potential of healthy cells loaded with JC-1 allows for the formation and sequestration of JC aggregates in the mitochondrion that is detected by a peak in red/orange fluorescence $(585 \mathrm{~nm})$ [15]. The various key events in apoptotic processes focus on the mitochondria, including the release of caspase activators (such as cytochrome $\mathrm{C}$ ), changes in electron transport, loss of mitochondrial transmembrane potential, and activation of lipid peroxidation processes in cells. The various signals that converge in the mitochondria to trigger or inhibit these events and their subsequent effects determine several major pathways of physiological cell death $[16,17]$.

\subsection{Effect of Fullerene C60 on Histomorphological Parameters} of CrFSTT of Rats. The histological structure in control samples of CrFSTT of rats was characterized by moderate damage according to the semiquantitative scale (Figure 4(a)). 
TABLe 1: Cytofluorimetric analysis of cells from CrFSTT after incubation with C60, staining with Annexin V and 7AAD, \% of cells.

\begin{tabular}{lccc}
\hline Sample/region & Annexin $\mathrm{V}^{-} / 7 \mathrm{AAD}^{-}$(live) & Annexin $\mathrm{V}^{+} / 7 \mathrm{AAD}^{-}$(apoptosis) & AnnexinV $^{+} / 7 \mathrm{AAD}^{+}+\mathrm{AnnexinV}^{-} / 7 \mathrm{AAD}^{+}(\mathrm{necrosis})$ \\
\hline Control & $69.31 \pm 1.27$ & $6.02 \pm 0.77$ & $24.67 \pm 1.12$ \\
C60_10 $\mu \mathrm{g} / \mathrm{mL}$ & $74.91 \pm 2.35$ & $5.92 \pm 0.54$ & $19.17 \pm 1.02$ \\
C60_15 $\mu \mathrm{g} / \mathrm{mL}$ & $79.86 \pm 1.24^{\mathrm{a}}$ & $4.28 \pm 1.18$ & $15.86 \pm 0.95^{\mathrm{a}}$ \\
C60_20 $\mu \mathrm{g} / \mathrm{mL}$ & $65.25 \pm 1.01^{\mathrm{b}}$ & $6.60 \pm 1.12$ & $28.15 \pm 1.08^{\mathrm{b}}$ \\
\hline
\end{tabular}

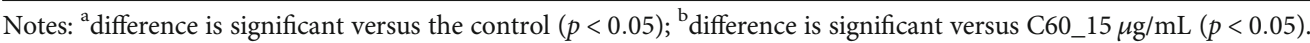

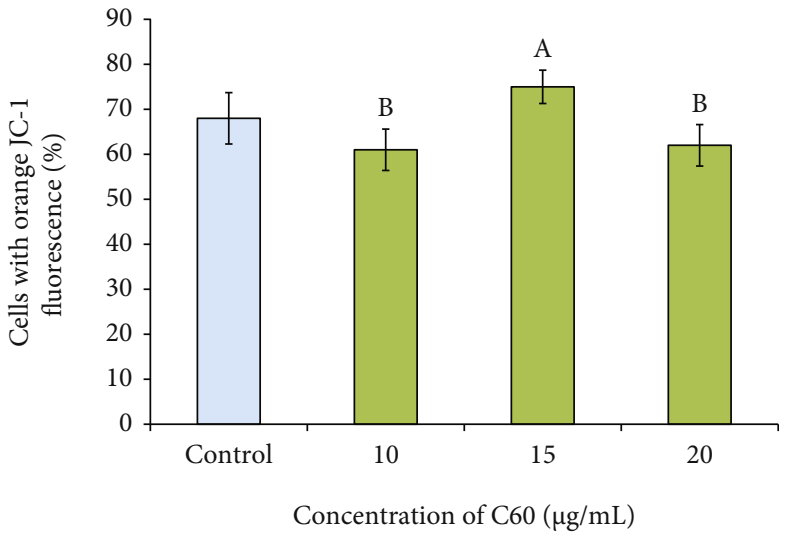

(a)

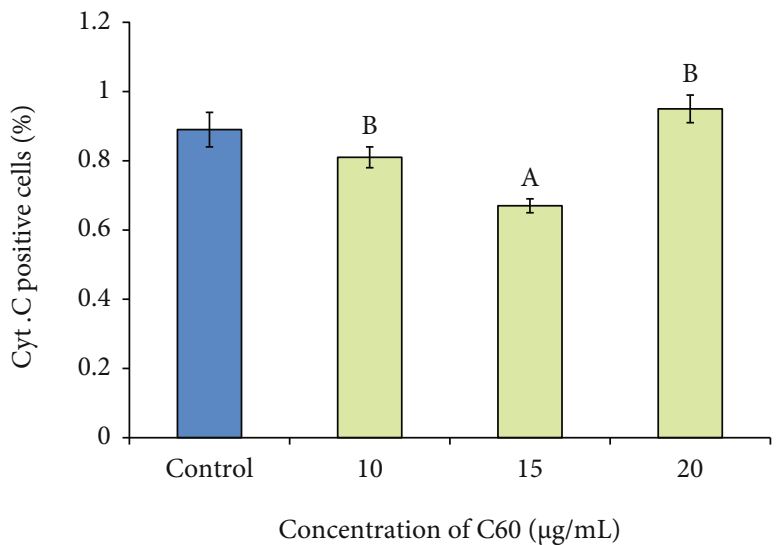

(b)

FIGURE 3: Mitochondrial activity (a) and level of cytochrome C release (b) in cells of CrFSTT after incubation with C60. Notes: ${ }^{\mathrm{A}}$ difference is significant versus the control $(p<0.05)$; ${ }^{\mathrm{B}}$ difference is significant versus C60_15 $\mu \mathrm{g} / \mathrm{mL}(p<0.05)$.
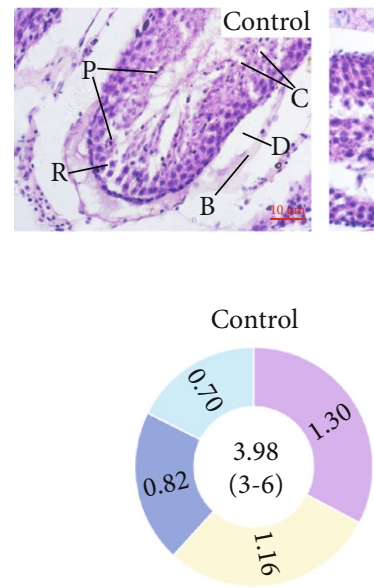

Cell retraction

Nuclei condensation
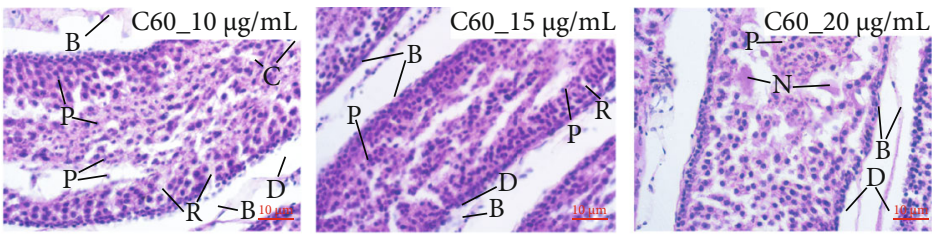

(a)

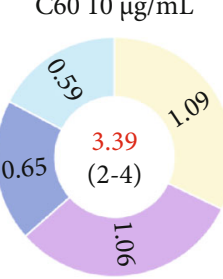

$\mathrm{C} 6015 \mu \mathrm{g} / \mathrm{mL}$

$\mathrm{C} 6020 \mu \mathrm{g} / \mathrm{mL}$
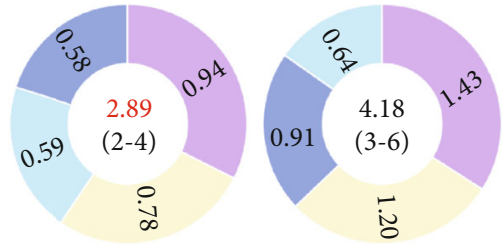

Cell detachment

Gap formation

(b)

FIGURE 4: CrFSTT of immature rats after incubation in the media with fullerene C60. (a) Light microscopy; staining with hematoxylin and eosin. Notes: B: basement membrane; C: cavities; D: desquamation of epithelium; N: necrosis; R: retraction of cells; P: pyknosis. (b) Morphological damage scoring of the spermatogenic epithelium. An average value of the total score is shown in the center of each diagram. Boundary values of the total points are specified in the round brackets. Values significantly different from control are highlighted in red $(p<0.05)$.

Basically, there was unexpressed cell retraction and insignificant spherical cavities in the epithelial layer and its partial desquamation. The basement membrane at the desquama- tion sites appeared thickened and swollen. In some of the germinogenic cells, especially in the adluminal compartment, pyknosis of the nuclei was noted. Incubation of 

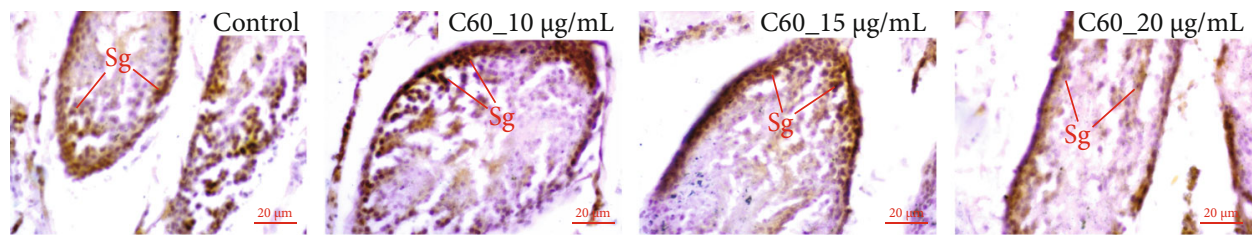

(a)

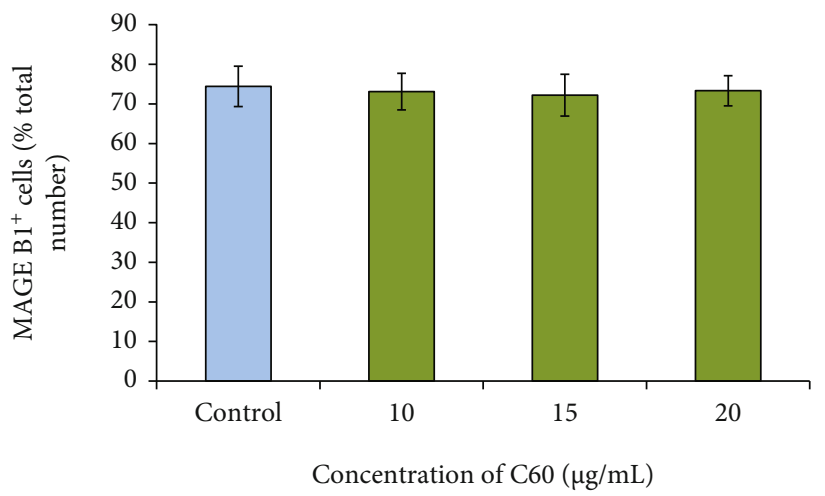

(b)

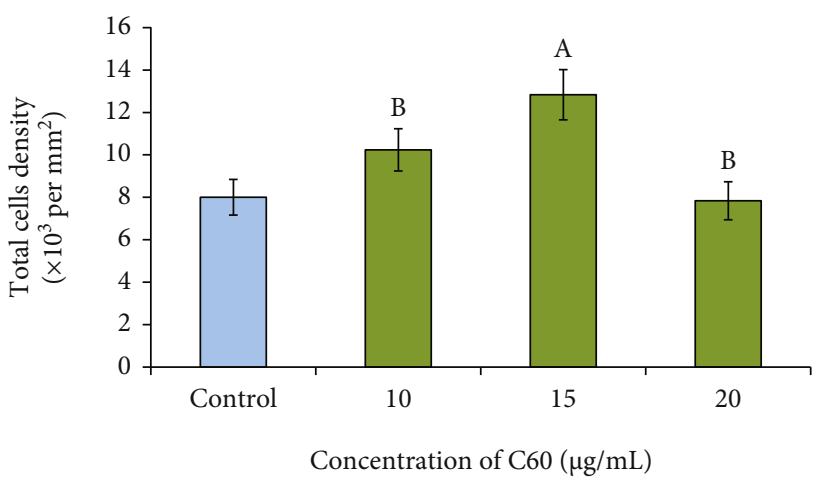

(c)

FIGURE 5: CrFSTT of immature rats after incubation in the media with fullerene C60. (a) Immunohistochemical staining for MAGE-B1, light microscopy. Notes: Sg: spermatogenic cells positively stained by MAGE-B1 (brown color); (b) relative amount of MAGE-B1 ${ }^{+}$cells; (c) average

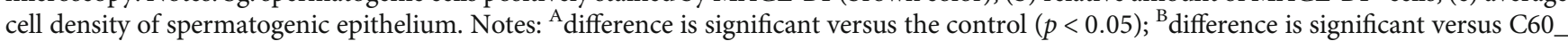
$15 \mu \mathrm{g} / \mathrm{mL}(p<0.05)$.

CrFSTT in the media with the addition of C60 at the concentrations of 10 and $15 \mu \mathrm{g} / \mathrm{mL}$ had a generally positive effect on the histostructure compared to the control.

The desquamation of the spermatogenic epithelium and swelling of the basement membrane were almost absent; the frequency of pyknosis of cell nuclei decreased with a more pronounced effect in the case of $15 \mu \mathrm{g} / \mathrm{mL}$ C60 addition (Figure 4(b)). The degree of the spermatogenic epithelium damage in this group decreased to 2.89 points, which corresponds to mild changes. The samples of CrFSTT incubated with $\mathrm{C} 60$ at a concentration of $20 \mu \mathrm{g} / \mathrm{mL}$ had a similar to control histological structure; only small foci of necrosis were observed in the central parts of the seminiferous tubules.

Cells positively stained for MAGE-B1 were located in a dense layer on the inner surface of the spermatogenic epithelium and had a rounded shape with a dense cytoplasm and a rounded nucleus (Figure 5(a)).

In histological sections of CrFSTT, it was possible to distinguish a population of typical spermatogenic cells which were positively stained by MAGE-B1. Stratification and disruption of the structural architecture of the tubules were observed; as a result of which the spermatogonia germ layer $\left(\mathrm{MAGE}-\mathrm{B} 1^{+}\right.$) was shifted to the center. Relative amount of MAGE-B1 ${ }^{+}$cells in CrFSTT did not change between the investigated media and ranged within $70-80 \%$ (Figure 5(b)). Thus, incubation with fullerene $\mathrm{C} 60$ did not have a selective effect on the population of MAGE-B $1^{+}$cells in the spermatogenic epithelium $(p<0.05)$.

According to the results of measuring of the average density of spermatogenic epithelial cells, it can be concluded that the addition of fullerene C60 to the rehabilitation medium for CrFSTT was effective at a concentration of $15 \mu \mathrm{g} / \mathrm{mL}$, sig- nificantly increasing this parameter relative to the control (by 1.6 times $(p<0.05))$ and other investigated concentrations (10 and $20 \mu \mathrm{g} / \mathrm{mL}$ ) (Figure 5(c)).

So, the histological examination showed a decrease of spermatogenic epithelium damage down to the mild degree and an increase of the average cell density of spermatogenic epithelium after incubation with $15 \mu \mathrm{g} / \mathrm{mL}$ C60. At the same time, it is important that the relationship between the spermatogenic epithelium and basement membrane was preserved because the last one in the testis serves as a reservoir of uniquely important cytokines to regulate junction dynamics that are associated with germ cell cycle progression and movement [18].

The use of modern cryopreservation technologies allows obtaining a stock of reproductive cells and tissues with their subsequent long-term storage at low temperatures, which allows them to be free transported and defrosted immediately before therapeutic use [19]. Determining the optimal conditions for cryopreservation of testicular tissue fragments is crucial for further restoration of fertility. It is known that freezing-thawing leads to a decrease in antioxidant protection of biological systems that causes the damage of the cell membrane integrity and activation of apoptosis and necrosis [20]. Free radicals initiate the processes of lipid peroxidation and inhibit mitochondrial enzymes of the respiratory chain, ATPase activity, etc. [21]. Interaction of highly reactive hydroxyl radicals with polyunsaturated fatty acids causes significant damage to cells and tissues, which in turn leads to increased permeability of cell membranes [22].

Thus, this research was aimed at comprehensively studying the effect of different concentrations of fullerene C60 on the antioxidant capacity, apoptotic/necrotic processes, and 
morphological characteristics of CrFSTT of immature rats. One of the important conditions for manifestation of the biological effect of fullerene C60 is their interaction with cell membranes. The $\mathrm{C} 60$ molecule is characterized by the hydrophobic properties and affinity to the biological membranes. Fullerenes can affect cellular membranes both through adsorption on the surface and through incorporation into the lipid bilayer [23]. The authors [24] reported that C60 in the concentration of $10^{-5} \mathrm{M}$ is able to penetrate the bilayer lipid membrane (phosphatidylcholine-cholesterol $(1: 1)$ ) locally increasing its conductivity. In addition, the authors [25] showed that molecules of fullerene C60 are connected predominantly with the mitochondria inside the cell. And this is very important because the mitochondria are very susceptible to oxidative damage due to large amounts of pathogenic mutant mtDNA can accumulate in the testis resulting mitochondrial respiratory dysfunction in spermatogenic cells that ultimately induces meiotic arrest and abnormalities in sperm morphology [26].

\section{Conclusions}

Our results demonstrated that 10 and $15 \mu \mathrm{g} / \mathrm{mL}$ C60 similarly affected TAS activity and ROS generation, but the use of fullerene C60 at concentration of $15 \mu \mathrm{g} / \mathrm{mL}$ led also to an increase in the activity of mitochondria, to a decrease in cytochrom $\mathrm{C}$ release, and to an increase in the cell density in histological section. Furthermore, all studied concentrations of fullerene C60 did not lead to significant changes in the level of processes of apoptosis in cells from CrFSTT of rats. At the same time, it should be noted that the percentage of living cells after incubation with $15 \mu \mathrm{g} / \mathrm{mL}$ C60 was higher and a value of necrotic ones was lower than in the control samples. In our study, the fullerene C60 regardless of concentration had no effect on the relative amount of MAGE-B1 ${ }^{+}$cells in CrFSTT of rats but at a concentration of $15 \mu \mathrm{g} / \mathrm{mL}$ reduced the degree of histological damage of spermatogenic epithelium. So, the general analysis of obtained data showed that the fullerene C60 can be used for rehabilitation of CrFSTT and it is the most optimal in the concentration of $15 \mu \mathrm{g} / \mathrm{mL}$. The results can be used for the development of an effective rehabilitation medium for CrFSTT using C60. These findings relate to the applied nanotechnology in its extension to reproductive medicine.

\section{Data Availability}

The data used to support the findings of this study are included within the article.

\section{Conflicts of Interest}

The authors declare that they have no competing interests.

\section{Authors' Contributions}

Anatoliy Goltsev and Nataliia Volkova contributed to the study design. Nataliia Volkova and Mariia Yukhta performed the experiments, analyzed the data, and wrote the manuscript. All authors read and approved the final manuscript.

\section{Acknowledgments}

This work was supported by the program of the National Academy of Sciences of Ukraine "Support for the development of priority areas of scientific research" (code 6541230), contract number 2.2.6.130.

\section{References}

[1] F. del Vento, M. Vermeulen, F. de Michele et al., "Tissue engineering to improve immature testicular tissue and cell transplantation outcomes: one step closer to fertility restoration for prepubertal boys exposed to gonadotoxic treatments," International Journal of Molecular Sciences, vol. 19, no. 1, p. 286, 2018.

[2] G. Bai, Z. Song, H. Geng et al., "Oxidized quasi-carbon nitride quantum dots inhibit ice growth," Advanced Materials, vol. 29, no. 28, p. 1606843, 2017.

[3] W. Rao, H. Huang, H. Wang et al., "Nanoparticle-mediated intracellular delivery enables cryopreservation of human adipose-derived stem cells using trehalose as the sole cryoprotectant," ACS Applied Materials \& Interfaces, vol. 7, no. 8, pp. 5017-5028, 2015.

[4] U. Ritter, Y. I. Prylutskyy, M. P. Evstigneev, N. A. Davidenko, and V. V. Cherepanov, "Structural features of highly stable reproducible $\mathrm{C} 60$ fullerene aqueous colloid solution probed by various techniques," Fullerenes, Nanotubes, and Carbon Nanostructures, vol. 23, no. 6, pp. 530-534, 2014.

[5] S. Emelyantsev, E. Prazdnova, V. Chistyakov, and I. Alperovich, "Biological effects of C60 fullerene revealed with bacterial biosensor-toxic or rather antioxidant?," Biosensors, vol. 9, no. 2, p. 81, 2019.

[6] S. Y. Zay, D. A. Zavodovskyi, K. I. Bogutska, D. N. Nozdrenko, and Y. I. Prylutskyy, "Prospects of C60 fullerene application as a mean of prevention and correction of ischemic-reperfusion injury in the skeletal muscle tissue," Fiziolohichny zhurnal, vol. 62, no. 3, pp. 66-77, 2016.

[7] D. M. Nozdrenko, D. O. Zavodovskyi, T. Y. Matvienko et al., "C60 fullerene as promising therapeutic agent for the prevention and correction of skeletal muscle functioning at ischemic injury," Nanoscale Research Letters, vol. 12, no. 1, p. 115, 2017.

[8] N. Volkova, M. Yukhta, and A. Goltsev, "Biopolymer gels as a basis of cryoprotective medium for testicular tissue of rats," Cell and Tissue Banking, vol. 19, no. 4, pp. 819-826, 2018.

[9] N. O. Volkova, M. S. Yukhta, L. G. Chernyshenko, L. V. Stepanyuk, L. V. Sokil, and A. M. Goltsev, "Cryopreservation of rat seminiferous tubules using biopolymers and slow noncontrolled rate cooling," Problems of Cryobiology and Cryomedicine, vol. 28, no. 4, pp. 278-292, 2018.

[10] P. Scharff, U. Ritter, O. P. Matyshevska et al., "Therapeutic reactive oxygen generation," Tumori Journal, vol. 94, no. 2, pp. 278-283, 2008.

[11] T. T. Turner and J. J. Lysiak, "Oxidative stress: a common factor in testicular dysfunction," Journal of Andrology, vol. 29, no. 5, pp. 488-498, 2008.

[12] R. Wu, J. Feng, Y. Yang et al., "Significance of serum total oxidant/antioxidant status in patients with colorectal cancer," PLoS One, vol. 12, no. 1, article e0170003, 2017. 
[13] O. O. Gonchar, A. V. Maznychenko, N. V. Bulgakova et al., "C60 Fullerene Prevents Restraint Stress-Induced Oxidative Disorders in Rat Tissues: Possible Involvement of the Nrf2/ARE-Antioxidant Pathway," Oxidative Medicine and Cellular Longevity, vol. 2018, Article ID 2518676, 17 pages, 2018.

[14] N. A. Volkova, M. S. Yukhta, E. V. Pavlovich, and A. N. Goltsev, "Change in functional state of bone marrow-derived mesenchymal stem cells after incubation with silver nanoparticles," in Nanophotonics, Nanooptics, Nanobiotechnology, and Their Applications, O. Fesenko and L. Yatsenko, Eds., pp. 273-282, Springer Nature, Cham, Switzerland, 2019.

[15] A. Cossarizza, M. Baccaranicontri, G. Kalashnikova, and C. Franceschi, "A New Method for the Cytofluorometric Analysis of Mitochondrial Membrane Potential Using the JAggregate Forming Lipophilic Cation 5,5',6,6' -Tetrachloro1,1',3,3' -tetraethylbenzimidazolcarbocyanine Iodide (JC-1)," Biochemical and Biophysical Research Communications, vol. 197, no. 1, pp. 40-45, 1993.

[16] R. A. Stuart, D. W. Nicholson, and W. Neupert, "Early steps in mitochondrial protein import: receptor functions can be substituted by the membrane insertion activity of apocytochrome c," Cell, vol. 60, no. 1, pp. 31-43, 1990.

[17] D. Sinha, "The complexities of human mitochondrial innermembrane protein translocases in the maintenance of organeller function," Proceedings of the Indian National Science Academy, vol. 83, no. 4, pp. 877-891, 2017.

[18] M. K. Siu and C. Y. Cheng, "Extracellular matrix: recent advances on its role in junction dynamics in the seminiferous epithelium during spermatogenesis," Biology of Reproduction, vol. 71, no. 2, pp. 375-391, 2004.

[19] Y. Song, R. Sharp, F. Lu, and M. Hassan, "The future potential of cryopreservation for assisted reproduction," Cryobiology, vol. 60, no. 3, pp. S60-S65, 2010.

[20] T. Finkel and N. J. Holbrook, "Oxidants, oxidative stress and the biology of ageing," Nature, vol. 408, no. 6809, pp. 239$247,2000$.

[21] M. Hermes-Lima and T. Zenteno-Savın, "Animal response to drastic changes in oxygen availability and physiological oxidative stress," Comparative Biochemistry and Physiology Part C: Toxicology \& Pharmacology., vol. 133, no. 4, pp. 537-556, 2002.

[22] R. Pamplona, "Membrane phospholipids, lipoxidative damage and molecular integrity: a causal role in aging and longevity," Biochimica et Biophysica Acta, vol. 1777, no. 10, pp. 12491262, 2008.

[23] A. Bianco, T. Da Ros, M. Prato, and C. Toniolo, "Fullerenebased amino acids and peptides," Journal of Peptide Science, vol. 7, no. 4, pp. 208-219, 2001.

[24] S. V. Prylutska, O. P. Matyshevska, I. I. Grynyuk, Y. I. Prylutskyy, U. Ritter, and P. Scharff, "Biological effects of C60 fullerenes in vitro and in a model system," Molecular Crystals and Liquid Crystals, vol. 468, no. 1, pp. 265/[617]-274/[626], 2007.

[25] S. Foley, C. Crowley, M. Smaihi et al., "Cellular localisation of a water-soluble fullerene derivative," Biochemical and Biophysical Research Communications, vol. 294, no. 1, pp. 116-119, 2002.

[26] S. Rajender, P. Rahul, and A. A. Mahdi, "Mitochondria, spermatogenesis and male infertility," Mitochondrion, vol. 10, no. 5, pp. 419-428, 2010. 